\title{
Some Properties of Soft $\alpha$-Open Sets in Soft Topological Space
}

\author{
${ }^{\mathrm{a}}$ Gnanambal Ilango, ${ }^{\mathrm{b}} \mathrm{B}$. Arun and ${ }^{\mathrm{c}} \mathrm{K}$. Saravana kumar \\ ${ }^{a, b, c}$ Department of Mathematics, Government Arts College (Autonomous), Coimbatore-641 018, Tamil Nadu,
} INDIA.

\begin{abstract}
In the present paper, soft $\alpha$-open and soft $\alpha$-closed sets in soft topological spaces are defined over an initial universe with a set of parameters. A necessary and sufficient condition for a soft set to be soft $\alpha$-open set in soft topological space is stated and proved. A detailed study is carried out on properties of soft $\alpha$-interier and soft $\alpha$-closure of soft sets.
\end{abstract}

Keywords: Soft $\alpha$-open sets, soft $\alpha$-closed sets, soft $\alpha$-interior, soft $\alpha$-closure.

\section{Introduction and Preliminaries}

Many disciplines in science and engineering depend on mathematical modeling. There are many tools available for reasoning and computing which are crisp, deterministic and precise in character.Also there are many complicated problems in economics, engineering, environment, medical science etc., which are not always crisp. So alternative theories are developed such as probability theory, fuzzy set theory etc., which can be considered as mathematical tool for dealing with uncertainties. However, all of these new theories have inherent difficulties. The reason for these difficulties is, possibly the inadequate parameterization tools of the theories.

In 1999, Molodtsov [3] initiated the concept of soft set theory as a mathematical tool for dealing with uncertainties which is free from the above difficulties. The notion of topological space for soft sets was formulated by Shabir et. al. [5], which is defined over an initial universe with a fixed set of parameters. Levine [1] introduced semi-open sets in topological spaces. Mahanta et. al. [2] introduced soft semi-open sets and soft semi-closed sets in soft topological spaces. Njastad [4] studied about $\alpha$-open sets in topological space in 1965. In this paper soft $\alpha$-open sets and soft $\alpha$-closed sets are defined in a soft topological space and some of their properties are studied.

Definition 1 ([3]). Let $U$ be an initial universal set and $E$ be the set of parameters. Let $P(U)$ denote the power set of $U$ and let $A \subseteq E$. A pair $(F, A)$ is called a soft set over $U$, where $F$ is a mapping given by $F: A \rightarrow P(U)$.

Definition 2([6]). A soft set $(F, A)$ over $U$ is said to be

(i) a null soft set, denoted by $\phi_{A}$, if $\forall e \in A, F(e)=\phi$.

(ii) an absolute soft set, denoted by $U_{A}$, if $\forall e \in A, F(e)=U$.

The soft sets $(F, A)$ over a universe $U$ in which all the parameters set $A$ are same is a family of soft sets denoted by $S S(U)_{A}$.

Soft operations are denoted by usual set theoretical operations with ' $\sim$ ' symbol above.

Proposition 1([6]). Let I be an arbitrary index set and $\left\{\left(F_{i}, A\right)\right\}_{i \in I}$ be a subfamily of $S S(U)_{A}$. Then

$$
\begin{aligned}
& \text { (i) }\left[\bigcup_{i \in I}^{\sim}\left(F_{i}, A\right)\right]^{c}=\bigcap_{i \in I}\left(F_{i}, A\right)^{c} \\
& \text { (ii) }\left[\bigcap_{i \in I}^{\tilde{N}}\left(F_{i}, A\right)\right]^{c}=\bigcup_{i \in I}\left(F_{i}, A\right)^{c}
\end{aligned}
$$

Definition 3([6]). Let $\tau$ be a collection of soft sets over a universe $U$ with a fixed set $A$ of parameters, then $\tau \subseteq S S(U)_{A}$ is called a soft topology on Uif,

(i) $\phi_{A}, U_{A}$ belong to $\tau$.

(ii) The union of any number of soft sets in $\tau$ belongs to $\tau$.

(iii) The intersection of any two soft sets in $\tau$ belongs to $\tau$.

The triplet $(U, \tau, A)$ is called soft topological space over $U$. The members of $\tau$ are called soft open sets in $U$ and complements of them are called soft closed sets over $U$.

Definition 4([6]). Let $(U, \tau, A)$ be a soft topological space and let $(G, A)$ be a soft set over $U$.

(i) The soft closure of $(G, A)$ is the soft set defined by

$$
\tilde{s} c l(G, A)=\widetilde{\cap}\{(S, A):(S, A) \text { is soft closed and }(G, A) \widetilde{\subseteq}(S, A)\}
$$

(ii) The soft interior of $(G, A)$ is the soft set defined by

$$
\tilde{\operatorname{sint}}(G, A)=\widetilde{\mathrm{U}}\{(S, A):(S, A) \text { is soft open and }(S, A) \widetilde{\subseteq}(G, A)\}
$$


Note that, $\tilde{s} \operatorname{cl}(G, A)$ is the smallest soft closed set containing $(G, A)$ and $\tilde{\operatorname{sint}}(G, A)$ is the largest soft open set contained in $(G, A)$.

Theorem 1([6]). Let $(U, \tau, A)$ be a soft topological space and let $(F, A)$ and $(G, A)$ be soft sets over U.Then,

(i) $\quad(F, A)$ is soft closed iff $(F, A)=\tilde{s} c l(F, A)$

(ii) $\quad(G, A)$ is soft open iff $(G, A)=\tilde{\operatorname{sint}}(G, A)$

Theorem 2([6]). Let $(U, \tau, A)$ be a soft topological space and let $(F, A)$ and $(G, A)$ be soft sets over $U$. Then,

(i) If $(F, A) \widetilde{\subseteq}(G, A)$ then $\tilde{s} c l(F, A) \widetilde{\subseteq} \tilde{s} c l(G, A)$

(ii) If $(F, A) \widetilde{\simeq}(G, A)$ then $\operatorname{sint}(F, A) \widetilde{\simeq} \operatorname{sint}(G, A)$

Remark 1. The following results hold good for soft topological spaces as in the case of topological spaces. If $\left\{(G, A)_{\alpha} \mid \alpha \in I\right\}$ is a collection of soft sets, then

(i) $\quad \tilde{\mathrm{U}} \tilde{s} \operatorname{cl}(G, A)_{\alpha} \widetilde{\subseteq} \tilde{s} c l\left(\widetilde{U}(G, A)_{\alpha}\right)$

(ii) $\tilde{U} \tilde{\operatorname{sint}}(G, A)_{\alpha} \widetilde{\operatorname{sint}}\left(\widetilde{U}(G, A)_{\alpha}\right)$

Definition 5([2]). In a soft topological space $(U, \tau, A)$, a soft set

(i) $\quad(G, A)$ is said to be softsemi-open set if $(G, A) \widetilde{\subseteq} \tilde{s} c l(\tilde{s i n t}(G, A))$

(ii) $\quad(F, A)$ is said to be soft semi-closed set if $(F, A) \supseteq \tilde{\operatorname{sint}}(\tilde{\operatorname{scl}}(F, A))$

\section{Soft $\alpha$-open sets and soft $\alpha$-closed sets}

In this section, we introduce soft $\alpha$-open and soft $\alpha$-closed sets are introduced and various properties of these sets are investigated.

Definition 6. In a soft topological space $(U, \tau, A)$, a soft set

(i) $(G, A)$ is said to be soft $\alpha$-open set if $(G, A) \widetilde{\widetilde{s i n t}}(\tilde{\operatorname{scl}}(\tilde{\operatorname{sint}}(G, A)))$

(ii) $(F, A)$ is said to be soft $\alpha$-closed set if $(F, A) \supseteq \tilde{s} \operatorname{cl}(\tilde{\operatorname{sint}}(\tilde{\operatorname{scl}}(F, A)))$

Example 1. Let $U=\left\{v_{1}, v_{2}, v_{3}\right\}, A=\left\{e_{1}, e_{2}, e_{3}\right\}$.

Let $F, G, H, I$ be the mappings from $A$ to $P(U)$ defined by,

$(F, A)=\left\{\left(e_{1}, U\right),\left(e_{2},\left\{v_{2}, v_{3}\right\}\right),\left(e_{3},\left\{v_{1}, v_{2}\right\}\right)\right\}$

$(G, A)=\left\{\left(e_{1}, \phi\right),\left(e_{2},\left\{v_{1}\right\}\right),\left(e_{3},\left\{v_{3}\right\}\right)\right\}$

$(H, A)=\left\{\left(e_{1},\left\{v_{2}\right\}\right),\left(e_{2},\left\{v_{1}, v_{3}\right\}\right),\left(e_{3},\left\{v_{3}\right\}\right)\right\}$

$(I, A)=\left\{\left(e_{1},\left\{v_{2}\right\}\right),\left(e_{2},\left\{v_{3}\right\}\right),\left(e_{3}, \phi\right)\right\}$. Then $(F, A),(G, A),(H, A)$ and $(I, A)$ are soft sets over $U$.

Then, $\tau=\{\tilde{\phi}, \widetilde{U},(F, A),(G, A),(H, A),(I, A)\}$ is a soft topology over $U$.

Hence $(U, \tau, A)$ is a soft topological space over $U$ and some of its

soft open sets are $\tilde{\phi}, \widetilde{U},(F, A),(G, A),(H, A),(I, A)$

soft closed sets are $\widetilde{U}, \tilde{\phi},\left(F^{c}, A\right),\left(G^{c}, A\right),\left(H^{c}, A\right),\left(I^{c}, A\right)$

where $\quad\left(F^{c}, A\right)=\left\{\left(e_{1}, \phi\right),\left(e_{2},\left\{v_{1}\right\}\right),\left(e_{3},\left\{v_{3}\right\}\right)\right\}=(G, A)$

$\left(G^{c}, A\right)=\left\{\left(e_{1}, U\right),\left(e_{2},\left\{v_{2}, v_{3}\right\}\right),\left(e_{3},\left\{v_{1}, v_{2}\right\}\right)\right\}=(F, A)$

$\left(H^{c}, A\right)=\left\{\left(e_{1},\left\{v_{1}, v_{3}\right\}\right),\left(e_{2},\left\{v_{2}\right\}\right),\left(e_{3},\left\{v_{1}, v_{2}\right\}\right)\right\}$

$\left.\left(I^{c}, A\right)=\left\{\left(e_{1},\left\{v_{1}, v_{3}\right\}\right),\left(e_{2},\left\{v_{1}, v_{2}\right\}\right),\left(e_{3}, U\right\}\right)\right\}$

Some soft semi-open sets of this soft topology are $(F, A),(G, A),(H, A),(I, A)$.

Note that $\left(H^{c}, A\right)$ and $\left(I^{C}, A\right)$ are not soft semi-open and

Some soft $\alpha$-open sets of this soft topology are $(F, A),(G, A),(H, A),(I, A)$.

Note that $\left(H^{c}, A\right)$ and $\left(I^{c}, A\right)$ are not soft $\alpha$-open.

Remark 2. $(\phi, A)$ and $(U, A)$ are always soft $\alpha$-open and soft $\alpha$-closed in a soft topological space $(U, \tau, A)$.

Theorem 3. (i) Every soft $\alpha$-open set is a soft semi-open set.

(ii) Every soft $\alpha$-closed set is a soft semi-closed set.

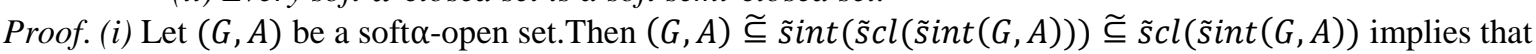
$(G, A)$ is soft semi-open.

(ii) Let $(F, A)$ be a soft $\alpha$-closed set.Then $(F, A) \supseteq \tilde{s} \operatorname{cl}(\tilde{\operatorname{sint}}(\tilde{\operatorname{s}} \operatorname{cl}(F, A))) \simeq \tilde{\operatorname{sint}}(\tilde{s} \operatorname{cl}(F, A))$ implies that $(F, A)$ is soft semi-closed.

The converse of the above theorem need not be true.

Theorem 4. $(G, A)$ is a soft $\alpha$-open set iff there exists a soft open set $(H, A)$ such that $(H, A) \widetilde{\subseteq}(G, A) \widetilde{\subseteq} \operatorname{sint}(\tilde{s} c l(H, A))$.

Proof. Suppose there exists a soft open set $(H, A) \operatorname{such}$ that $(H, A) \widetilde{\subseteq}(G, A) \widetilde{\subseteq} \operatorname{sint}(\tilde{s} c l(H, A))$.

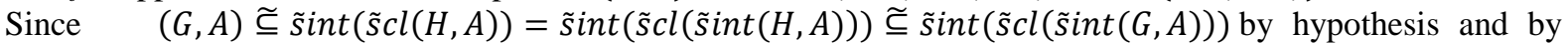
Theorem 2(ii), $(G, A)$ is soft $\alpha$-open.

On the other hand, Let $(G, A)$ be soft $\alpha$-open.Then $(G, A) \widetilde{\subseteq} \operatorname{sint}(\tilde{s} \operatorname{cl}(\tilde{\operatorname{sint}}(G, A)))$

Let $\tilde{\operatorname{sint}}(G, A)=(H, A)$. Since $\tilde{\operatorname{sint}}(G, A) \widetilde{\subseteq}(G, A),(H, A) \widetilde{\subseteq}(G, A)$ and also $(G, A) \widetilde{\subseteq} \tilde{\operatorname{sint}}(\tilde{s} c l(H, A))$. Hence there exists a soft open set $(H, A)$ such that $(H, A) \widetilde{\subseteq}(G, A) \widetilde{\subseteq} \operatorname{sint}(\tilde{s} c l(H, A))$.

Theorem 5. $(F, A)$ is a soft $\alpha$-closed set iff there exists a soft closed set $(K, A)$ such that $\tilde{s} \operatorname{cl}(\tilde{\operatorname{sint}}(K, A)) \widetilde{\subseteq}(F, A) \widetilde{\subseteq}(K, A)$. 
Proof. $\quad$ Let $(F, A)$ be soft $\alpha$-closed.Then $\tilde{\operatorname{scl}}(\tilde{\operatorname{sint}}(\tilde{\operatorname{s}} \mathrm{cl}(F, A))) \widetilde{\subseteq}(F, A)$

Let $\tilde{s} c l(F, A)=(K, A)$. Then $(K, A)$ is soft closed.

Since $(F, A) \widetilde{\subseteq} \tilde{s} c l(F, A),(F, A) \widetilde{\subseteq}(K, A)$ and $\tilde{\operatorname{scl}}(\tilde{\operatorname{sint}}(\tilde{s} c l(F, A))) \widetilde{\subseteq}(F, A) \Rightarrow \tilde{s} c l(\tilde{\operatorname{sint}}(K, A)) \widetilde{\subseteq}(F, A)$.

Thus there exists a soft closed set $(K, A)$ such that $\tilde{\operatorname{scl}}(\tilde{\operatorname{sint}}(K, A)) \widetilde{\subseteq}(F, A) \widetilde{\subseteq}(K, A)$.

On the other hand, suppose there exists a soft closed set $(K, A)$ such that $\tilde{s} \operatorname{cl}(\tilde{\operatorname{sint}}(K, A)) \widetilde{\subseteq}(F, A) \widetilde{\subseteq}(K, A)$

Since $(K, A)$ is soft closed, $\tilde{s} c l(K, A)=(K, A)$, By hypothesis, $\tilde{\operatorname{scl}}(\tilde{\operatorname{sint}}(K, A)) \widetilde{\subseteq}(F, A)$

$\Rightarrow \tilde{s} c l(\tilde{\operatorname{sint}}(\tilde{s} \operatorname{cl}(K, A))) \widetilde{\subseteq}(F, A) .(F, A) \widetilde{\subseteq}(K, A) \Rightarrow \tilde{s} \operatorname{cl}(F, A) \widetilde{\subseteq} \operatorname{scl}(K, A) \Rightarrow$

$\tilde{\operatorname{scl}}(\tilde{\operatorname{sint}}(\tilde{\operatorname{s} c l}(F, A))) \widetilde{\widetilde{s}} \operatorname{cl}(\tilde{\operatorname{sint}}(\tilde{\operatorname{s} c l}(K, A))) \widetilde{\subseteq}(F, A) \Rightarrow(F, A)$ is soft $\alpha$-closed.

Theorem 6. Arbitrary union of soft $\alpha$-open sets is a soft $\alpha$-open set.

Proof. Let $\left\{(G, A)_{\lambda} \mid \lambda \in \mathrm{I}\right\}$ be a collection of soft $\alpha$-open sets of a soft topological space $(U, \tau, A)$. Then there

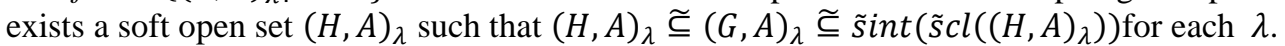

Hence, $\quad \widetilde{U}(H, A)_{\lambda} \widetilde{\subseteq} \widetilde{U}(G, A)_{\lambda} \widetilde{\subseteq} \widetilde{U}\left(\tilde{s i n t}\left(\tilde{s} c l(H, A)_{\lambda}\right)\right) \widetilde{\subseteq} \operatorname{sint}\left(\tilde{s} c l\left(\widetilde{U}(H, A)_{\lambda}\right)\right) \quad($ by $\quad$ Remark $\quad 1) \Rightarrow$

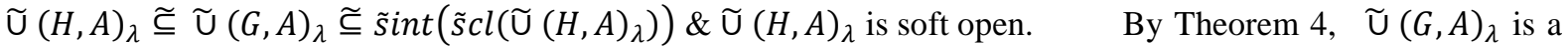
soft $\alpha$-open set.

Theorem 7. Arbitrary intersection of soft $\alpha$-closed sets is a soft $\alpha$-closed set.

Proof. Let $\left\{(G, A)_{\lambda} \mid \lambda \in \mathrm{I}\right\}$ be a collection of soft $\alpha$-closed set of a soft topological space $(U, \tau, A)$.Then $\left\{\left((G, A)_{\lambda}\right)^{c} \mid \lambda \in \mathrm{I}\right\}$ is a collection of soft $\alpha$-open sets. By Theorem $6, \widetilde{\mathrm{U}}\left((G, A)_{\lambda}\right)^{c}$ is a soft $\alpha$-open set.That is $\left(\widetilde{\cap}(G, A)_{\lambda}\right)^{c}$ is a soft $\alpha$-open set, by Preposition 1. Hence, $\widetilde{\cap}(G, A)_{\lambda}$ is a soft $\alpha$-closed set.

We shall denote the family of all soft $\alpha$-open sets (soft $\alpha$-closed sets) of a soft topological space $(U, \tau, A)$ by $\alpha-O S S(U)_{A}\left(\alpha-\operatorname{CSS}(U)_{A}\right)$.

Definition 7. Let $(U, \tau, A)$ be a soft topological space and $(G, A)$ be a soft set over $U$.

(i) The soft $\alpha$-closure of $(G, A)$ is a soft set defined by

$\tilde{s} \alpha-c l(G, A)=\widetilde{\cap}\left\{(S, A):(G, A) \widetilde{\subseteq}(S, A)\right.$ and $\left.(S, A) \in \alpha-C S S(U)_{A}\right\}$

(ii) The soft $\alpha$-interior of $(G, A)$ is a soft set defined by

$\tilde{s} \alpha-\operatorname{int}(G, A)=\widetilde{U}\left\{(S, A):(S, A) \widetilde{\subseteq}(G, A)\right.$ and $\left.(S, A) \in \alpha-O S S(U)_{A}\right\}$

Note that, $\tilde{s} \alpha-\operatorname{cl}(G, A)$ is the smallest soft $\alpha$-closed set containing $(G, A)$ and $\tilde{s} \alpha$-int $(G, A)$ is the largest soft $\alpha$-open set contained in $(G, A)$.

Theorem 8. Let $(U, \tau, A)$ be a soft topological space and $(G, A)$ be a soft set over $U$. Then,

(i) $(G, A) \in \alpha-\operatorname{CSS}(U)_{A} i f f(G, A)=\tilde{s} \alpha-\operatorname{cl}(G, A)$

(ii) $(G, A) \in \alpha-O S S(U)_{A}$ iff $(G, A)=\tilde{s} \alpha-\operatorname{int}(G, A)$

(iii) $\tilde{s} \alpha-\operatorname{cl}(\tilde{s} \alpha-\operatorname{cl}(G, A))=\tilde{s} \alpha-\operatorname{cl}(G, A)$

(iv) $\tilde{s} \alpha-\operatorname{int}(\tilde{s} \alpha-\operatorname{int}(G, A))=\tilde{s} \alpha-\operatorname{int}(G, A)$

(v) $(\tilde{s} \alpha-\operatorname{cl}(G, A))^{c}=\tilde{s} \alpha-\operatorname{int}\left(G^{c}, A\right)$

(vi) $(\tilde{s} \alpha-\operatorname{int}(G, A))^{c}=\tilde{s} \alpha-c l\left(G^{c}, A\right)$

Proof. Let $(G, A)$ be a soft set over $U$.

(i) Let $(G, A)$ be a soft $\alpha$-closed set. Then it is the smallest soft $\alpha$-closed set containing itself, so $(G, A)=\tilde{s} \alpha-c l(G, A)$ by definition.

On the other hand, suppose $(G, A)=\tilde{s} \alpha-\operatorname{cl}(G, A) . \tilde{s} \alpha-\operatorname{cl}(G, A)$ being the intersection of soft $\alpha$-closed sets is soft $\alpha$-closed, sos $\alpha$-cl $(G, A) \in \alpha-\operatorname{CSS}(U)_{A} \Rightarrow(G, A) \in \alpha-\operatorname{CSS}(U)_{A}$

(ii) Let $(G, A)$ be a soft $\alpha$-open set. Then it is the largest soft $\alpha$-open set contained in $(G, A)$. By $\operatorname{definition}(G, A)=\tilde{s} \alpha-\operatorname{int}(G, A)$

On the other hand, suppose $(G, A)=\tilde{s} \alpha$-int $(G, A) \cdot \tilde{s} \alpha$-int $(G, A)$ being the union of soft $\alpha$-open sets is soft $\alpha$-open, so $\tilde{\operatorname{s}} \alpha$-int $(G, A) \in \alpha$-OSS $(U)_{A} \Rightarrow(G, A) \in \alpha-O S S(U)_{A}$

(iii) $\quad \tilde{s} \alpha-\operatorname{cl}(G, A) \in \alpha-\operatorname{CSS}(U)_{A}$ by definition of soft closure and Theorem 7,

$\tilde{s} \alpha-\operatorname{cl}(\tilde{s} \alpha-\operatorname{cl}(G, A))=\tilde{s} \alpha-\operatorname{cl}(G, A)$ by $(i)$

(iv) $\quad \tilde{s} \alpha-\operatorname{int}(G, A) \in \alpha-O S S(U)_{A}$ by definition of soft interior and Theorem 6, $\tilde{s} \alpha-\operatorname{int}(\tilde{s} \alpha-\operatorname{int}(G, A))=\tilde{s} \alpha-\operatorname{int}(G, A)$ by $(i i)$

(v) $\quad(\tilde{s} \alpha-c l(G, A))^{c}=\left(\widetilde{\cap}\left\{(S, A):(G, A) \widetilde{\subseteq}(S, A) \&(S, A) \in \alpha-C S S(U)_{A}\right\}\right)^{c}$ $=\widetilde{U}\left\{(S, A)^{c}:(G, A)^{c} \cong(S, A)^{c} \&(S, A)^{c} \in \alpha-O S S(U)_{A}\right\}$ $=\widetilde{U}\left\{\left(S^{c}, A\right):\left(S^{c}, A\right) \widetilde{\subseteq}\left(G^{c}, A\right) \&\left(S^{c}, A\right) \in \alpha-\operatorname{OSS}(U)_{A}\right\}$ $=\tilde{s} \alpha$-int $\left(G^{c}, A\right)$

(vi) $\quad(\tilde{s} \alpha-\operatorname{int}(G, A))^{c}=\left(\widetilde{\cup}\left\{(S, A):(S, A) \widetilde{\subseteq}(G, A) \&(S, A) \in \alpha-O S S(U)_{A}\right\}\right)^{c}$ $=\widetilde{\cap}\left\{(S, A)^{c}:(S, A)^{c} \cong(G, A)^{c} \&(S, A)^{c} \in \alpha-\operatorname{CSS}(U)_{A}\right\}$ $=\widetilde{\cap}\left\{\left(S^{c}, A\right):\left(G^{c}, A\right) \widetilde{\subseteq}\left(S^{c}, A\right) \&\left(S^{c}, A\right) \in \alpha-\operatorname{CSS}(U)_{A}\right\}$ $=\tilde{s} \alpha-c l\left(G^{c}, A\right)$

Corollary 1. Let $(U, \tau, A)$ be a soft topological space then

(i) $\tilde{s} \alpha-c l(\phi, A)=(\phi, A)$ and $\tilde{s} \alpha-c l(U, A)=(U, A)$

(ii) $\tilde{s} \alpha-\operatorname{int}(\phi, A)=(\phi, A)$ and $\tilde{s} \alpha-\operatorname{int}(U, A)=(U, A)$ 
Proof. (i) Since $(\phi, A)$ and $(U, A)$ are soft $\alpha$-closed sets, by Theorem 8(i),

$\tilde{s} \alpha-c l(\phi, A)=(\phi, A)$ and $\tilde{s} \alpha-c l(U, A)=(U, A)$

(ii) Since $(\phi, A)$ and $(U, A)$ are soft $\alpha$-open sets, by Theorem 8(ii),

$\tilde{s} \alpha$-int $(\phi, A)=(\phi, A)$ and $\tilde{s} \alpha$-int $(U, A)=(U, A)$

Theorem 9. Let $(U, \tau, A)$ be a soft topological space and $(G, A)$ and $(K, A)$ be two soft sets over $U$. Then,

(i) $(G, A) \widetilde{\subseteq}(K, A) \Rightarrow \tilde{s} \alpha-\operatorname{int}(G, A) \widetilde{\simeq} \tilde{s} \alpha-\operatorname{int}(K, A)$

(ii) $(G, A) \widetilde{\subseteq}(K, A) \Rightarrow \tilde{s} \alpha-\operatorname{cl}(G, A) \widetilde{\simeq} \tilde{s} \alpha-\operatorname{cl}(K, A)$

(iii) $\tilde{s} \alpha-\operatorname{cl}((G, A) \widetilde{U}(K, A))=\tilde{s} \alpha-c l(G, A) \widetilde{U} \tilde{s} \alpha-\operatorname{cl}(K, A)$

(iv) $\tilde{s} \alpha-\operatorname{int}((G, A) \widetilde{\cap}(K, A))=\tilde{s} \alpha-\operatorname{int}(G, A) \widetilde{n} \tilde{s} \alpha-\operatorname{int}(K, A)$

(v) $\tilde{s} \alpha-\operatorname{cl}((G, A) \widetilde{\cap}(K, A)) \widetilde{\sim} \tilde{s} \alpha-c l(G, A) \widetilde{\cap} \tilde{s} \alpha-\operatorname{cl}(K, A)$

(vi) $\tilde{s} \alpha-\operatorname{int}((G, A) \widetilde{\cup}(K, A)) \widetilde{s} \tilde{s} \alpha-\operatorname{int}(G, A) \widetilde{U} \tilde{s} \alpha-\operatorname{int}(K, A)$

Proof. Let $(G, A)$ and $(K, A)$ be two soft sets over $U$.

(i) By definition of soft $\alpha$-interior,

$\tilde{s} \alpha-\operatorname{int}(G, A)=\widetilde{U}\left\{(S, A):(S, A) \widetilde{\subseteq}(G, A) \&(S, A) \in \alpha-O S S(U)_{A}\right\} \&$

$\tilde{s} \alpha-\operatorname{int}(K, A)=\widetilde{U}\left\{(T, A):(T, A) \widetilde{\subseteq}(K, A) \&(T, A) \in \alpha-O S S(U)_{A}\right\}$

Now, $\tilde{s} \alpha-\operatorname{int}(G, A) \widetilde{\subseteq}(G, A) \widetilde{\subseteq}(K, A) \Rightarrow \tilde{s} \alpha-\operatorname{int}(G, A) \widetilde{\subseteq}(K, A)$

Since $\tilde{s} \alpha$-int $(K, A)$ is the largest soft $\alpha$-open set contained in $(K, A)$

Therefore, $\tilde{s} \alpha-\operatorname{int}(G, A) \widetilde{\subseteq} \tilde{s} \alpha-\operatorname{int}(K, A)$

(iii) By definition of soft $\alpha$-closure,

$\tilde{s} \alpha-\operatorname{cl}(G, A)=\widetilde{\cap}\left\{(S, A):(G, A) \widetilde{\subseteq}(S, A) \&(S, A) \in \alpha-\operatorname{CSS}(U)_{A}\right\} \&$

$\tilde{s} \alpha-\operatorname{cl}(K, A)=\widetilde{\cap}\left\{(T, A):(K, A) \widetilde{\subseteq}(T, A) \&(T, A) \in \alpha-\operatorname{CSS}(U)_{A}\right\}$

since $(G, A) \widetilde{\subseteq} \tilde{s} \alpha-c l(G, A)$ and $(K, A) \widetilde{\subseteq} \tilde{s} \alpha-c l(K, A)$

$\Rightarrow(G, A) \widetilde{\subseteq}(K, A) \widetilde{\subseteq} \tilde{s} \alpha-c l(K, A) \Rightarrow(G, A) \widetilde{\subseteq} \tilde{s} \alpha-c l(K, A)$

But $\tilde{s} \alpha-\operatorname{cl}(G, A)$ is the smallest soft semi-closed set containing $(G, A)$

Therefore, $\tilde{s} \alpha-c l(G, A) \widetilde{\simeq} \tilde{s} \alpha-c l(K, A)$.

(iii) Since, $(G, A) \widetilde{\subset}(G, A) \widetilde{\cup}(K, A)$ and $(K, A) \widetilde{\subset}(G, A) \widetilde{\cup}(K, A)$

By $(i i), \tilde{s} \alpha-c l(G, A) \widetilde{\sim} \tilde{s} \alpha-c l((G, A) \widetilde{\cup}(K, A))$ and

$\tilde{s} \alpha-c l(K, A) \widetilde{\sim} \tilde{s} \alpha-c l((G, A) \widetilde{U}(K, A))$

$\Rightarrow \tilde{s} \alpha-\operatorname{cl}(G, A) \widetilde{\mathrm{U}} \tilde{s} \alpha-\operatorname{cl}(K, A) \widetilde{\sim} \tilde{s} \alpha-\operatorname{cl}((G, A) \widetilde{U}(K, A))$

Now, $\tilde{s} \alpha-c l(G, A), \tilde{s} \alpha-c l(K, A) \in \alpha-\operatorname{CSS}(U)_{A}$

$\Rightarrow \tilde{s} \alpha-c l(G, A) \widetilde{U} \tilde{s} \alpha-\operatorname{cl}(K, A) \in \alpha-C S S(U)_{A}$

Then $(G, A) \widetilde{\subset} \tilde{s} \alpha-c l(G, A)$ and $(K, A) \widetilde{\subset} \tilde{s} \alpha-c l(K, A)$

$\Rightarrow(G, A) \widetilde{\cup}(K, A) \widetilde{\subset} \tilde{s} \alpha-c l(G, A) \widetilde{\cup} \tilde{s} \alpha-c l(K, A)$

i.e., $\tilde{s} \alpha-c l(G, A) \widetilde{U} \tilde{s} \alpha-c l(K, A)$ is a soft $\alpha$-closed set containing $(G, A) \widetilde{U}(K, A)$. But,

$\tilde{s} \alpha-\operatorname{cl}((G, A) \widetilde{\cup}(K, A))$ is the smallest soft $\alpha$-closed set containing $(G, A) \widetilde{\cup}(K, A)$.

Hence $\tilde{s} \alpha-c l((G, A) \widetilde{\cup}(K, A)) \widetilde{\sim} \tilde{s} \alpha-\operatorname{cl}(G, A) \widetilde{\cup} \tilde{s} \alpha-c l(K, A) \quad \ldots(2)$

From (1) \& (2), $\tilde{s} \alpha-\operatorname{cl}((G, A) \widetilde{U}(K, A))=\tilde{s} \alpha-\operatorname{cl}(G, A) \widetilde{\cup} \tilde{s} \alpha-\operatorname{cl}(K, A)$.

(iv) $\quad$ Since $(G, A) \widetilde{\cap}(K, A) \widetilde{\subset}(G, A)$ and $(G, A) \widetilde{\cap}(K, A) \widetilde{\subset}(K, A)$

By $(i), \tilde{s} \alpha-i n t((G, A) \widetilde{\cap}(K, A)) \widetilde{\subset} \tilde{s} \alpha-i n t(G, A)$ and

$\tilde{s} \alpha-\operatorname{int}((G, A) \widetilde{\cap}(K, A)) \widetilde{c} \tilde{s} \alpha-\operatorname{int}(K, A)$

$\Rightarrow \tilde{s} \alpha-\operatorname{int}((G, A) \widetilde{\cap}(K, A)) \widetilde{\sim} \tilde{s} \alpha-\operatorname{int}(G, A) \widetilde{\cap} \tilde{s} \alpha-\operatorname{int}(K, A)$

Now, $\tilde{\sin } \alpha-\operatorname{int}(G, A), \tilde{s} \alpha-\operatorname{int}(K, A) \in \alpha-\operatorname{OSS}(U)_{A}$

$\Rightarrow \tilde{s} \alpha-\operatorname{int}(G, A) \widetilde{\cap} \tilde{s} \alpha-\operatorname{int}(K, A) \in \alpha-O S S(U)_{A}$

Then $\tilde{s} \alpha-\operatorname{int}(G, A) \widetilde{\subset}(G, A)$ and $\tilde{s} \alpha-\operatorname{int}(K, A) \widetilde{\subset}(K, A)$

$\Rightarrow \tilde{s} \alpha-\operatorname{int}(G, A) \widetilde{\cap} \tilde{s} \alpha-\operatorname{int}(K, A) \widetilde{\subset}(G, A) \widetilde{\cap}(K, A)$

i.e., $\tilde{s} \alpha$-int $(G, A) \widetilde{\cap} \tilde{s} \alpha$-int $(K, A)$ is a soft $\alpha$-open set contained in $(G, A) \widetilde{\cap}(K, A)$. But,

$\tilde{s} \alpha$-int $((G, A) \widetilde{\cap}(K, A))$ is the largest soft $\alpha$-open set contained in $(G, A) \widetilde{\cap}(K, A)$.

Hence $\tilde{s} \alpha$-int $(G, A) \widetilde{n} \tilde{s} \alpha-\operatorname{int}(K, A) \widetilde{\subset} \tilde{s} \alpha-\operatorname{int}((G, A) \widetilde{\cap}(K, A)) \ldots(4)$

From (3) \& (4), s̃ $\alpha-\operatorname{int}((G, A) \widetilde{\cap}(K, A))=\tilde{s} \alpha-\operatorname{int}(G, A) \widetilde{\cap} \tilde{s} \alpha-i n t(K, A)$.

(v) Since, $(G, A) \widetilde{\cap}(K, A) \widetilde{\sim}(G, A)$ and $(G, A) \widetilde{\cap}(K, A) \widetilde{\subset}(K, A)$

$\Rightarrow \tilde{s} \alpha-c l((G, A) \widetilde{\cap}(K, A)) \widetilde{\sim} \tilde{s} \alpha-c l(G, A)$ and

$\tilde{s} \alpha-\operatorname{cl}((G, A) \widetilde{\cap}(K, A)) \widetilde{\sim} \tilde{s} \alpha-c l(K, A)$

$\Rightarrow \tilde{s} \alpha-c l((G, A) \widetilde{\cap}(K, A)) \widetilde{\sim} \tilde{s} \alpha-c l(G, A) \widetilde{\cap} \tilde{s} \alpha-c l(K, A)$

(vi) Since, $(G, A) \widetilde{\subset}(G, A) \widetilde{U}(K, A)$ and $(K, A) \widetilde{\subset}(G, A) \widetilde{U}(K, A)$

$\Rightarrow \tilde{s} \alpha-\operatorname{int}(G, A) \widetilde{\subset} \tilde{s} \alpha-\operatorname{int}((G, A) \widetilde{\cup}(K, A))$ and

$\tilde{s} \alpha-\operatorname{int}(K, A) \widetilde{\sim} \tilde{s} \alpha-\operatorname{int}((G, A) \widetilde{U}(K, A))$

$\Rightarrow \tilde{s} \alpha-\operatorname{int}(G, A) \widetilde{\cup} \tilde{s} \alpha-\operatorname{int}(K, A) \widetilde{\sim} \tilde{s} \alpha-\operatorname{int}((G, A) \widetilde{\cup}(K, A))$ 


\section{Conclusion}

The soft $\alpha$-open sets and soft $\alpha$-closed sets defined here have many properties similar to soft semi-open and soft semi-closed sets respectively. It is interesting to note that arbitrary union of soft $\alpha$-open sets are soft $\alpha$-open. Soft interior and soft closure are defined like interior and closure of topological spaces and many of their properties are studied.

\section{References}

[1] N. Levine, Semi-open sets and semi-continuity in topological spaces, Amer. Math. Monthly, Vol. 70 (1963), 36-41.

[2] J. Mahanta and P. K. Das, On soft topological space via semi-open and semi-closed soft sets, arXiv [math.GN.], Vol. 1(2012), 1-9.

[3] D. Molodtsov, Soft set theory-First results, Comput. Math.Appli., Vol. 37 (1999), 19-31.

[4] O. Njastad, On some classes of nearly open sets, Pacific Journal of Mathematics, Vol. 15, No. 3, (1965), 961-970.

[5] M. Shabir and M. Naz, On soft topological spaces, Comput. Math.Appli., Vol. 61 (2011), 1786-1799.

[6] I. Zorlutuna, M. Akdag, W. K. Min and S. Atmaca, Remarks on soft topological spaces, Annals of Fuzzy Mathematics and Informatics, Vol. 3, No. 2 (2012), 171-185. 